\title{
Transcript Abundance of Phytoene Synthase 1 and Phytoene Synthase 2 Is Associated with Natural Variation of Storage Root Carotenoid Pigmentation in Carrot
}

\author{
Megan J. Bowman' \\ Plant Breeding and Plant Genetics Program, University of Wisconsin-Madison, 1575 Linden Drive, \\ Madison, WI 53706 \\ David K. Willis \\ U.S. Department of Agriculture, Agricultural Research Service, Vegetable Crops Research Unit and \\ Department of Plant Pathology, University of Wisconsin-Madison, 1630 Linden Drive, Madison, \\ WI 53706 \\ Philipp W. Simon ${ }^{2}$
U.S. Department of Agriculture, Agricultural Research Service, Vegetable Crops Research Unit and
Department of Horticulture, University of Wisconsin-Madison, 1575 Linden Drive, Madison,
WI 53706
}

AdDITIONAL INDEX words. Daucus carota, gene expression, naturally occurring mutations, RT-qPCR

\begin{abstract}
Carotenoids are isoprenoid compounds synthesized in plants that serve as photoprotectants essential for photosynthesis and provide plant tissues with red, orange, and yellow pigmentation. These compounds are important in human health, because they serve as both vitamin A precursors as well as having antioxidant properties. Carrot (Daucus carota ssp. sativus) provides an important source of carotenoids in the human diet, providing up to $30 \%$ of provitamin A in the United States. Although essential to human health, very little is currently understood about the accumulation of carotenoids in carrot. To better understand the molecular mechanism for carotenoid accumulation in carrot, we used reverse-transcription quantitative polymerase chain reaction (PCR) to evaluate the expression of nine genes in the carotenoid biosynthetic pathway in storage root tissue. No significant difference was found among white, yellow, orange, and dark orange carrot roots in seven of the nine genes evaluated. However, increased phytoene synthase 1 (PSY1) and phytoene synthase 2 (PSY2) expression was observed in orange and dark orange carrot roots compared with yellow and white carrots. Increased PSY1 and PSY2 expression was not observed in the leaf tissue of these genotypes, indicating a different mechanism for carotenoid accumulation in the leaf tissue of carrot. This study is the first to demonstrate that naturally occurring mutations that dramatically increase carotenoid accumulation in orange carrot are associated with increased PSY1 and PSY2 expression and it provides insights into the mechanism underlying the biosynthesis of these important photoprotectants and nutrients.
\end{abstract}

Differential expression of genes in the carotenoid biosynthesis pathway serves as a regulatory mechanism for carotenoid accumulation in diverse plant species such as tomato [Solanum lycopersicum (Fray and Grierson, 1993; Ronen et al., 2000)], maize [Zea mays (Buckner et al., 1996; Harjes et al., 2008; Vallabhaneni and Wurtzel, 2009)], and marigold [Tagetes erecta (Moehs et al., 2001)]. Of these previously studied plant species, none contributes as much to overall provitamin A carotenoid consumption in the U.S. diet as carrots (Simon et al., 2009). Despite its importance, the molecular regulation of carotenoid accumulation in the storage root of carrot has not been extensively researched.

Received for publication 25 Mar. 2013. Accepted for publication 6 Dec. 2013. M.J.B. was supported by the Gabelman-Seminis Distinguished Graduate Research Fellowship at the University of Wisconsin-Madison and P.W.S. received USDA Specialty Crop Research Initiative award 2008-51180-04896. We thank Douglas Senalik for his assistance with the high-performance liquid chromatography experiment and data analysis.

${ }^{1}$ Current address: Department of Plant Biology, Michigan State University, 612 Wilson Road, East Lansing, MI 48824.

${ }^{2}$ Corresponding author. E-mail: Philipp.Simon@ars.usda.gov.
The genetic basis of carotenoid accumulation in carrot has been studied and genomic sequences of 24 carotenoid biosynthesis genes in carrot have been published (Just et al., 2007). Among these are genes synthesizing upstream precursors to carotenoid biosynthesis, enzymes directly related to carotenoid biosynthesis, and downstream carotenoid catabolic enzymes. Using primers from published carrot sequence and degenerate primers from other plant species, $5^{\prime}$ rapid amplification of cDNA ends PCR was used to isolate the $5^{\prime}$ regions of cDNAs, and the full-length coding regions of each of these genes were sequenced. Twenty-two of these carotenoid biosynthesis-related genes were placed on the linkage map of a cross between the U.S. Department of Agriculture (USDA) orange carrot inbred line B493 and whiterooted Queen Anne's lace (Daucus carota ssp. carota) or wild carrot (Just et al., 2007). The cDNA sequence and the subsequent mapping of carotenoid biosynthesis genes in carrot provide an important foundation to further examine and understand the regulation of carotenoid accumulation in carrot storage roots.

Interestingly, preliminary evidence of carotenoid gene expression in non-pigmented carrot root by reverse transcriptase 
PCR (Just, 2004) provided the first evidence that all genes in the carotenoid biosynthetic pathway were expressed in carrot roots of all colors including white carrots where no carotenoids accumulate. It was unexpected that the complex biosynthetic machinery for carotenoid biosynthesis would be transcribed in tissue that accumulates no carotenoids. This led us to the hypothesis that differential expression of one or more genes in that pathway may account for, or at least contribute to, the large differential accumulation of carotenoids observed in comparing white and orange carrots.

The analysis of differential carotenoid gene expression during the development of orange, red, yellow, and white carrot storage roots has also been previously studied using reverse transcription quantitative PCR (RT-qPCR) (Clotault et al., 2008). Measuring gene expression at several time points in development up to 3 months post-planting, the genes PSY1, PSY2, phytoene

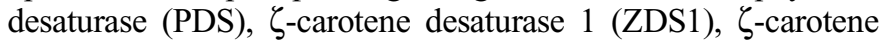
desaturase 2 (ZDS2), lycopene $\varepsilon$-cyclase (LCYE), lycopene $\beta$-cyclase (LCYB), and zeaxanthin epoxidase (ZEP) were analyzed using RT-qPCR. With the exception of LCYE in the luteinaccumulating yellow cultivar Yellowstone, and ZDS1in the lycopene-accumulating red cultivar Nutrired (Clotault et al., 2008), most genes were not differentially expressed in this study and none of the eight carotenoid biosynthesis genes evaluated were differentially expressed in comparing orange and white carrots. Although the results of Clotault et al. (2008) provided a quantitative analysis of carotenoid gene expression in carrot, their experimental design used pooled transcripts, thereby eliminating the ability to detect biological variation among samples. Furthermore, their statistical analyses used to normalize $\mathrm{Ct}$ values (now designated as quantitative cycle or $\mathrm{Cq}$ values) may have reduced the sensitivity of their analysis.

With these limitations in mind, we evaluated white, yellow, orange, and dark orange carrots following the now established guidelines for RT-qPCR, the "Minimum Information for Publication Quantitative Real-time PCR Experiments" or MIQE guidelines (Bustin et al., 2009). These guidelines focus on extraction protocols, transcript quality, reference gene accuracy, and publication standards necessary for high-quality RT-qPCR research. The objective of this study is to evaluate the expression of nine genes in the carotenoid biosynthetic pathway in storage root tissue of white, yellow, orange, and dark orange carrots. We present evidence for differential levels of transcript abundance for the PSY1 and PSY2 genes, which we interpret as differential expression of these carotenoid biosynthesis genes, in a comparison of carrot germplasm with diverse levels of carotenoid accumulation ranging from white to dark orange. The differential expression of PSY1 and PSY2 suggests that regulation of carotenoid biosynthesis early in the biochemical pathway alters carotene accumulation in the storage root of carrot, and these expression differences are associated with naturally occurring $Y$ and $Y_{2}$ color mutations previously identified in carrot (Just et al., 2009).

\section{Materials and Methods}

Plant materials. Germplasm representing a wide range of pigmented carrot material was used in both the RT-qPCR and high-performance liquid chromatography (HPLC) experiments. White cultivated (B6644), yellow (B7248), orange (B2566), dark orange (B2327) experimental inbreds developed by the USDA carrot breeding program were grown for each genotype under greenhouse conditions. Plants were harvested at 11 and 14 weeks post-planting, a time relatively early in color development when adequate tissue volume for RNA extraction is available. Four carrots per genotype were selected arbitrarily from of each color class at harvest. Root and leaf tissues sampled for both RNA and carotene analyses were flash frozen in liquid nitrogen and stored at $-80{ }^{\circ} \mathrm{C}$.

RNA EXTRACTION AND CDNA SYNTHESIS. From each carrot color class, $1 \mathrm{~g}$ of root tissue from four individual carrots was homogenized in liquid nitrogen and total RNA was extracted using the RNeasy kit (Qiagen,Valencia, CA) using the manufacturer's protocol. DNA was removed using the TurboDNAfree kit (Life Technologies, Grand Island, NY) using the rigorous protocol. RNA was quantified using a spectrophotometer (NanoDrop; ThermoFisher Scientific, Waltham, MA) and quality examined using the Bioanalyzer 2100 (Agilent, Santa Clara, CA). A no-reverse transcription control was used to determine DNA contamination using the actin reference gene (X17526.1). RNA samples with $\mathrm{Cq}$ values below 32 were considered contaminated with DNA. The concentration of RNA for each cDNA reaction was normalized to the level of the lowest concentration within all samples. cDNAs were synthesized from total carrot RNA with the iScript kit (Bio-Rad Laboratories, Hercules, CA) using the manufacturer's specifications. cDNA was diluted 10-fold for use in RTqPCR reactions. Synthesized cDNAs were stored at $-20{ }^{\circ} \mathrm{C}$.

Primer efficiencies. Primers were designed using the Beacon Designer software with the SYBRGreen module (Premier Biosoft International, Palo Alto, CA) (Table 1) and diluted to a concentration of $5 \mu \mathrm{M}$. Template DNA was obtained through PCR using the primers specifically designed for qPCR. PCR products were purified from agarose gel using a silica DNA purification protocol (Continental Laboratory Products, San Diego, CA). Two independent 10-fold dilutions of DNA were subjected to RT-qPCR using SYBR Green reaction mix (Bio-Rad Laboratories). Master mixes from each dilution series were split into three reactions $(25 \mu \mathrm{L}$ per well) yielding six replicate reactions for each dilution point. RT-qPCR was performed using the MyiQ Real-Time PCR Detection System with the standard two-step amp + melt protocol and analyzed using the MyiQ software package (Bio-Rad Laboratories). Efficiencies were calculated as the slope of the standard curve using the formula $\mathrm{E}=10^{(-1 / \mathrm{slope})}$ (Pfaffl, 2001; Rasmussen, 2001). Primer efficiencies between 1.8 and 2.0 were deemed acceptable for further use.

ReVerse TRANSCRIPTION QPCR. Briefly, we used Maxima SYBR Green qPCR Master Mix (ThermoFisher Scientific) and the iCycler Real Time PCR Detection System (Bio-Rad Laboratories). Reactions were performed in duplicate $25-\mu \mathrm{L}$ volumes with cDNA from two independent cDNA iScript reactions analyzed. The default two-step amplification plus melt curve protocol was used for each reaction (Bio-Rad Laboratories). Target transcripts included mRNA from nine genes in the carotenoid biosynthetic pathway (Table 1). Carrot actin (GenBank no. X17526.1) and ubiquitin (GenBank no. U68751.1) genes were selected as reference transcripts and validated using the BestKeeper program (Pfaffl et al., 2004) for transcript stability in each of the four carrot genotypes. Relative expression ratios (RERs) were calculated using the $\Delta \mathrm{Ct}$ method (Pfaffl, 2001) using mRNA levels in orange carrot as the calibrator for all target genes. RT-qPCR protocols followed the MIQE guidelines in RNA extraction, assessing template 


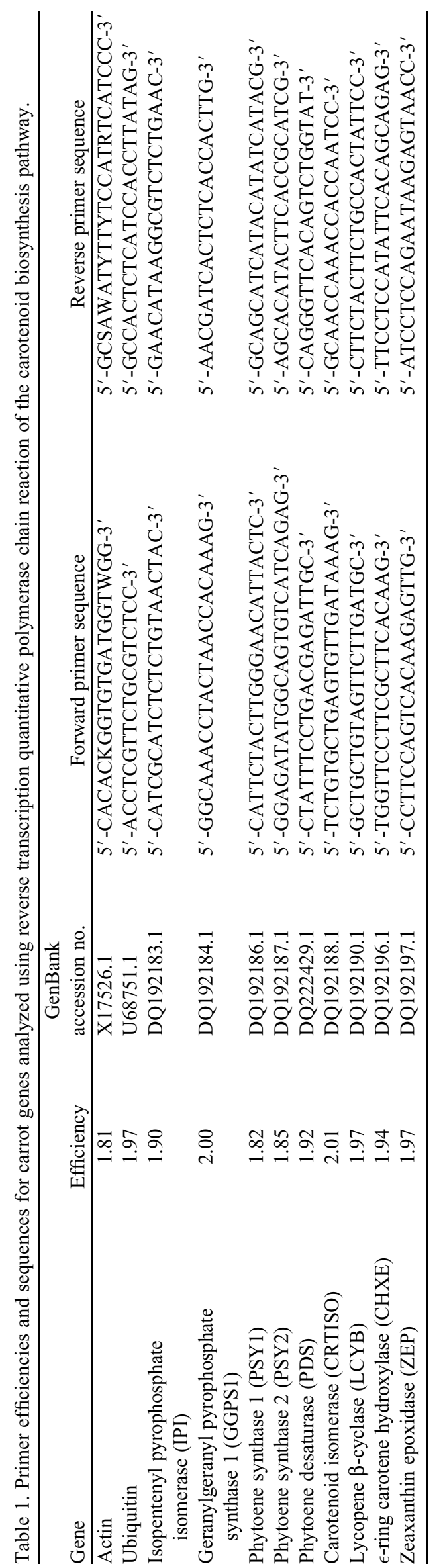

quality, experimental design, and data analysis (Bustin et al., 2009).

Carotene extraction and HPLC analysis. One gram of frozen tissue was taken from six plants for each genotype when RNA was extracted, homogenized with liquid nitrogen, and lyophilized for $4 \mathrm{~d}$. Tissue samples were split into two $0.03 \mathrm{-g}$ aliquots of dried root tissue and taken from each biological replicate to account for extraction variance and then extracted in $2 \mathrm{~mL}$ of hexane. Extractions were carried out overnight at $20{ }^{\circ} \mathrm{C} ; 700 \mu \mathrm{L}$ of methanol was added to $300 \mu \mathrm{L}$ of carotene extract and filtered extracts were analyzed with HPLC using a Rainin Microsorb-MV column (Mettler-Toledo, Columbus, $\mathrm{OH}$ ) using the previously published protocol (Just et al., 2007; Khachik et al., 1992; Simon and Wolff, 1987). Carotenoid concentrations were calculated as micrograms per gram on a fresh weight basis.

Statistics. Contingent on significant genotype effects from analysis of variance (ANOVA), the Tukey's post hoc test was used to determine differences between genotypes for both mean RER for transcript abundance from RT-qPCR analysis, and for content of $\alpha$-carotene, $\beta$-carotene, phytoene, lycopene, and lutein from HPLC $(\alpha=0.05)$. All statistical analyses were performed using the $\mathrm{R}$ statistics program ( $\mathrm{R}$ Development Core Team, 2011).

\section{Results}

RT-qPCR was used to quantify transcripts and infer the expression level of nine genes in the carotenoid biosynthetic pathway in storage roots of 11-week-old carrots when roots were $10 \%$ to $20 \%$ of full size and root color was relatively well developed. All genes analyzed in the carotenoid biosynthetic pathway were expressed in white carrot, where very little or no carotenoid pigment accumulates. In fact, no significant difference in transcript abundance was detected among white, yellow, orange, or dark orange roots for the isopentenyl pyrophosphate isomerase [IPI (GenBank no. DQ192183.1)] and geranylgeranyl pyrophosphate synthase 1 [GGPS1 (GenBank no. DQ192184.1)] genes early in the pathway or for most genes later in the pathway, including PDS (GenBank no. DQ222429.1), carotenoid isomerase [CRTISO (GenBank no. DQ192188.1)], $\varepsilon$-ring carotene hydroxylase [CHXE (GenBank no. DQ192196.1)], LCYB (GenBank no. DQ192190.1), and ZEP (GenBank no. DQ192197.1) (data not presented).

In contrast to these genes, expression of PSY 1 (GenBank no. DQ192186.1) was 5.0-fold greater in both orange (B2566) and dark orange (B2327) carrots than in white carrots (B6644) (Fig. 1) with an intermediate level of expression in yellow carrots (B7248). Furthermore, the expression of PSY2 (GenBank no. DQ192187.1) was greater in dark orange carrots than in orange, yellow, or white carrots by a factor of 2.3 to 4.3 $(P=0.05)$. Similar differential expression patterns for PSY1 and PSY2 were found across the diverse color classes at 14 weeks post-planting (Fig. 1). In contrast, neither PSY1 nor PSY2 nor any of the other genes evaluated were differentially expressed in the leaf tissue comparing of white, yellow, orange, and dark orange storage root genotypes (data not presented).

White cultivated carrot storage roots contained only trace (less than $1 \mu \mathrm{g} \cdot \mathrm{g}^{-1}$ ) levels of carotenoid pigments; yellow roots contained predominantly lutein with small amounts of $\beta$-carotene and trace levels of $\alpha$-carotene and phytoene; and both orange and dark orange carrots contained predominantly $\alpha$-carotene and 

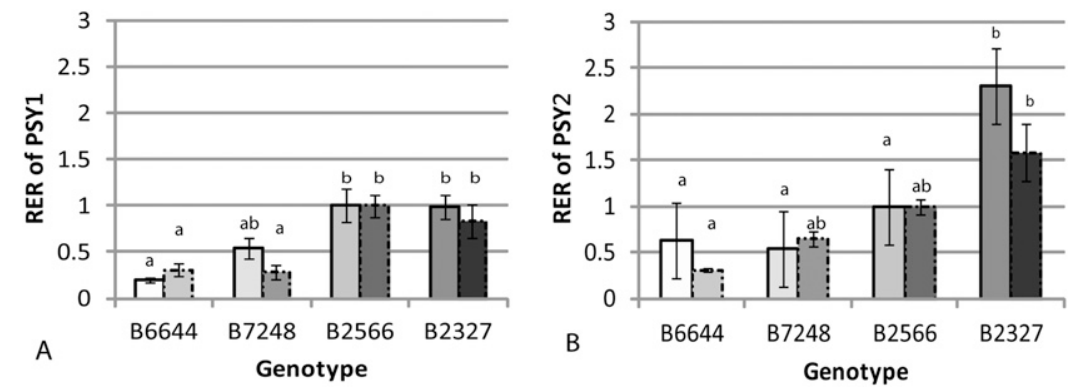

Fig. 1. Relative expression ratios (RERs) based up reverse transcription quantitative polymerase chain reaction (RT-qPCR) analysis of phytoene synthase 1 (PSY1) (A) and phytoene synthase 2 (PSY2) (B) genes at 11 weeks post-planting (solid lines) and 14 weeks post-planting (dashed lines) in carrot genotypes B6644 (white storage roots), B7248 (yellow), B2566 (orange), and B2327 (dark orange). Values for a given post planting evaluation with different letters indicate significant differences between genotypes as determined by analysis of variance and Tukey's post hoc test for each time point. Error bars represent SE of genotype means.

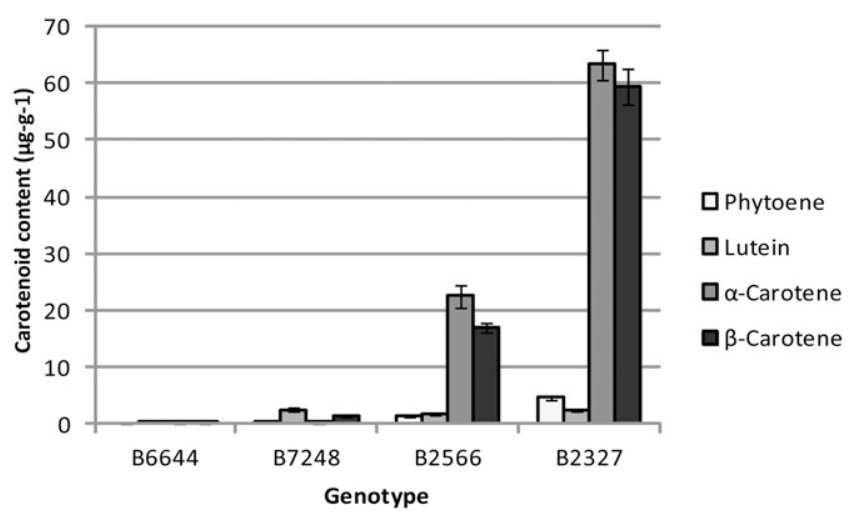

Fig. 2. Content of carotenoids phytoene, lutein, $\alpha$-carotene, and $\beta$-carotene in the storage roots of carrot genotypes B6644 (white storage roots), B7248 (yellow), B2566 (orange), and B2327 (dark orange) based on high-performance liquid chromatography at 11 weeks post-planting. Error bars represent SE of genotype means.

$\beta$-carotene with relatively little lutein and phytoene detected (Fig. 2). Dark orange carrot roots contained 2.8- to 3.5-fold more $\alpha$-carotene, $\beta$-carotene, and phytoene and 1.4-fold more lutein than orange carrots (Fig. 2). Lutein content was similar in yellow, orange, and dark orange carrots, and phytoene content never exceeded $5 \mu \mathrm{g} \cdot \mathrm{g}^{-1}$ in any genotype. None of the carrots evaluated contained lycopene. Total carotenoid content (the sum of the aforementioned pigments) varied widely among genotypes ranging from a trace in white carrots to $4 \mu \mathrm{g} \cdot \mathrm{g}^{-1}$ in yellow carrots to 42 and $130 \mu \mathrm{g} \cdot \mathrm{g}^{-1}$ in orange and dark orange carrots (Fig. 2; Table 2). Leaf total carotenoid content ranged from 63 to $80 \mu \mathrm{g} \cdot \mathrm{g}^{-1}$ across genotypes with no significant genotype effect in ANOVA (Table 2).

\section{Discussion}

Carotenoid biosynthetic genes have previously been associated with pigment phenotypes in tomato including LCYB (Ronen et al., 2000), CRTISO (Isaacson et al., 2002), and ZEP (Thorup et al., 2000). In contrast, RT-qPCR analysis of the carotenoid biosynthetic pathway in carrot has demonstrated that most of the genes analyzed in the carotenoid pathway are not differentially expressed in carrot genotypes that vary dramatically in carotenoid pigmentation. Genes evaluated include the early pathway genes GGPS1 and IPI. IPI catalyzes the conversion of isopentenyl pyrophosphate to dimethylallyl pyrophosphate (DMAPP), which is an important component to the synthesis of many diverse plant metabolites, including carotenoids, tocopherols, and chlorophylls (Okada et al., 2008). CHXE was included in this study because it was identified in previous research (Just et al., 2007) as being a potential candidate gene controlling pigment accumulation in carrot based on structural gene proximity to the $Y_{2}$ gene on the genetic map but was not differentially expressed in this study. Although the accumulation of carotenoids in the storage root of carrot provides no obvious function to the plant, downstream metabolites of the carotenoid pathway include apocarotenoids, like strigolactones and abscisic acid, which are important regulators of plant development. Given the importance of DMAPP and downstream metabolites, perhaps it should not be surprising that genes in the carotenoid pathway are expressed in carrot storage roots regardless of root color. Furthermore, observations made in other plants would suggest that research efforts should be placed on quantifying these downstream compounds in the storage root of carrot (Auldridge et al., 2006; Cazzonelli, 2011; Vallabhaneni et al., 2010).

Although most genes analyzed in this study exhibited no differential expression between carrot storage roots that varied widely in carotenoid content, the transcripts of two genes of significant importance were more abundant in orange- and dark orange-rooted carrot genotypes. As the first steps committed to the carotenoid biosynthetic pathway, PSY1 and PSY2 have a key role in the biosynthesis of carotenoid pigments in all plant species. As presented in this research, RT-qPCR has detected the differential expression of PSY1 and PSY2, as determined by relative transcript abundance, in comparing white, orange, and dark orange carrot storage root tissue. Phytoene synthase catalyzes the reaction converting geranylgeranyl pyrophosphate to phytoene, the first substrate toward the synthesis of carotenoids. Mutations in phytoene synthase have been responsible for a variety of pigment mutants in different plant species, including the $r$ mutant in tomato (Fray and Grierson, 1993), c2 in pepper [Capsicum annum (Huh et al., 2001)], and $y 1$ in maize (Buckner et al., 1996). Therefore, it is not surprising that differential expression of these genes in carrot accounts for at least part of the differential pigment accumulation in the storage root tissue.

Previous analysis of the carotenoid biosynthesis pathway of carrot (Santos et al., 2005) has also suggested an important role for phytoene synthase in regulating carotenoid accumulation in a carrot population segregating for white $\left(Y_{Y} Y_{2} Y_{2}\right)$, yellow $\left(y y Y_{2} Y_{2}\right)$, and orange $\left(y y y_{2} y_{2}\right)$ root color. It must be noted that although PSY1 and PSY2 are the only two carotenoid biosynthetic genes that vary among white $\left(Y_{Y} Y_{2} Y_{2}\right)$, yellow $\left(y y Y_{2} Y_{2}\right)$, and orange $\left(y y y_{2} y_{2}\right)$ roots, these genes are not candidates for $Y$ and $Y_{2}$. The magnitude of variation of gene expression between white and dark orange carrots is significant (Fig. 1) but small relative to differences in total carotene content, which varies from 0 to $121 \mu \mathrm{g} \cdot \mathrm{g}^{-1}$ among these contrasting genotypes (Fig. 2). Furthermore, neither $Y$ nor $Y_{2}$ is linked to PSY1 or PSY2 (Just et al., 2007). 
Table 2. Mean total carotenoid content (sum of phytoene, lutein, $\alpha$-carotene, and $\beta$-carotene) in the storage roots and leaves of carrot genotypes B6644 (white storage roots), B7248 (yellow), B2566 (orange), and B2327 (dark orange) based on high-performance liquid chromatography at 11 and 14 weeks post-planting.

\begin{tabular}{|c|c|c|c|c|c|c|c|c|c|}
\hline & Plant age post-planting & B6644 & & B7248 & & B2566 & & B2327 & \\
\hline Tissue & (weeks) & $\left(\mu \mathrm{g} \cdot \mathrm{g}^{-1}\right)$ & $\mathrm{SE}$ & $\left(\mu \mathrm{g} \cdot \mathrm{g}^{-1}\right)$ & SE & $\left(\mu \mathrm{g} \cdot \mathrm{g}^{-1}\right)$ & $\mathrm{SE}$ & $\left(\mu \mathrm{g} \cdot \mathrm{g}^{-1}\right)$ & $\mathrm{SE}$ \\
\hline Root & 11 & 0 & 0 & 4 & 1 & 42 & 3 & 130 & 5 \\
\hline Root & 14 & 0 & 0 & 10 & 2 & 112 & 12 & 260 & 26 \\
\hline
\end{tabular}

Although not genetically linked to the carotenoid pigment loci $Y$ and $Y_{2}$, further research efforts are ongoing to elucidate potential regulatory mechanisms of PSY1 and PSY2 expression in carrot. Research underway includes full genomic sequence analysis of PSY1 and PSY2 coding and promoter regions to identify any significant differences that could lead to differential expression in white- and orange-pigmented root tissue. PSY1 and PSY2 were not differentially expressed and pigment accumulation did not vary significantly in the leaf tissue of the inbred lines with white, orange, yellow, and dark orange storage root tissue. This is not unexpected, because low accumulation of carotenoids within leaf tissue, where they serve as essential photoprotectants, would likely disrupt photosynthesis.

The role of differential gene expression during the development of carrot storage roots has been previously investigated (Clotault et al., 2008), but in contrast to our study, the expression of PSY1 and PSY2 was not found to be different in white, yellow, and orange roots. The use of different genetic stocks, which included dark orange carrots, changes in plant propagation and different data analyses as discussed earlier, may account for the contrasting results of our studies.

The study of carotenoid gene expression and function has also been previously evaluated in transgenic carrot. Maass et al. (2009) used transgenes to demonstrate that the functional overexpression of bacterial phytoene synthase in non-green carrot callus caused the sequestration of carotenoids in crystals throughout the callus tissue and confirmed an increase of crystalline carotenoid structures within the cells of the transformed wild, white carrot roots. This is similar to what is found in orange carrot root tissue (Ben-Shaul and Klein, 1965), where carotenoids are found in crystalline form throughout storage root cells. The Maass et al. (2009) study further confirmed the importance of phytoene synthase in the accumulation of carotenoids in carrot by quantifying phytoene synthase protein amounts in varied carrot germplasm as well as overexpressing $\operatorname{crt} B$, a bacterial phytoene synthase gene in white carrot root tissue, where carotenoids, specifically $\beta$ carotene, accumulated in significant amounts. This study also suggested that within photosynthetic tissue, there is regulation that limits the accumulation of carotenoids, where they serve as photoprotectants quenching free radicals created during photosynthesis.

These findings of Maass et al. (2009), combined with those presented in this study suggest a significant but previously unidentified importance for PSY1 and PSY2 expression in carrot. However, these studies do not, by themselves, demonstrate that the 2- to 5-fold increased abundance of PSY1 and PSY2 transcripts establishes a threshold of gene expression adequate to account for the dramatic accumulation in carotenoids conditioned by $y$ and $y_{2}$ in orange and dark orange carrots. It is possible that the accumulation of the pigments within crystalline structures in the carrot storage root is regulated outside of the carotenoid pathway itself. For example, accumulation of carotenoids may be controlled by the development of chromoplasts, non-chlorophyll-containing plastids, as found for the $\mathrm{Or}$ mutation found in cauliflower [Brassica oleracea var. botrytis $(\mathrm{Li}$ et al., 2001, 2003, 2006; Li and Garvin, 2003; Li and Van Eck, 2007; Lu et al., 2006)]. As discussed in Cazzonelli and Pogson (2010), potential sources of regulation of carotenoid accumulation in plants include the development of plastids to serve as sinks to capture the carotenoids pigments in plant tissue. Further research will be necessary to understand the relationship between plastid development and the dramatic variation in accumulation of carotenoid pigments across simply inherited carrot color mutants. Other sources of regulation discussed include potential epigenetic and metabolic feedback of carotenoids, and those are new areas of research to be pursued in carrot, a vegetable crop of great importance in the human diet. To accomplish this goal, it will be necessary to move from a candidate gene evaluation of carotenoid biosynthesis to whole genome applications such as microarray, high-throughput genome, and RNA sequencing to understand more about this complicated process in plant metabolism.

\section{Literature Cited}

Auldridge, M., D.R. McCarty, and H.J. Klee. 2006. Plant carotenoid cleavage oxygenases and their apocarotenoid products. Curr. Opin. Plant Biol. 9:315-321.

Ben-Shaul, Y. and S. Klein. 1965. Development and structure of carotene bodies in carrot roots. Bot. Gaz. 126:79-85.

Buckner, B., P. Miguel, D. Janick-Buckner, and J . Bennetzen. 1996. The $Y 1$ gene of maize codes for phytoene synthase. Genetics 143:479-488.

Bustin, S.A., V. Benes, J.A. Garson, J. Hellemans, J. Huggett, M. Kubista, R. Mueller, T. Nolan, M.W. Pfaffl, G.L. Shipley, J. Vandespompele, and C.T. Wittwer. 2009. The MIQE guidelines: Minimum information for publication of quantitative real-time PCR experiments. Clin. Chem. 55:611-622.

Cazzonelli, C.I. 2011. Carotenoids in nature: Insights from plants and beyond. Funct. Plant Biol. 38:833-847.

Cazzonelli, C.I. and B.J. Pogson. 2010. Source to sink: Regulation of carotenoid biosynthesis in plants. Trends Plant Sci. 15:266-274.

Clotault, J., D. Peltier, R. Berruyer, M. Thomas, M. Briard, and E. Geoffriau. 2008. Expression of carotenoid biosynthesis genes during carrot root development. J. Expt. Bot. 59:3563-3573.

Fray, R. and D. Grierson. 1993. Identification and genetic-analysis of normal and mutant phytoene synthase genes of tomato by sequencing, complementation and co-suppression. Plant Mol. Biol. 22:589-602.

Harjes, C.E., T.R. Rocheford, L. Bai, T.P. Brutnell, C.B. Kandianis, S.G. Sowinski, A.E. Stapleton, R. Vallabhaneni, M. Williams, E.T . Wurtzel, J . Yan, and E.S . Buckler. 2008. Natural genetic variation in lycopene epsilon cyclase tapped for maize biofortification. Science 319:330-333.

Huh, J.H., B.C. Kang, S.H. Nahm, S. Kim, K.S. Ha, M.H. Lee, and B.D. Kim. 2001. A candidate gene approach identified phytoene 
synthase as the locus for mature fruit color in red pepper (Capsicum spp.). Theor. Appl. Genet. 102:524-530.

Isaacson, T., G. Ronen, D. Zamir, and J. Hirschberg. 2002. Cloning of tangerine from tomato reveals a carotenoid isomerase essential for the production of $\beta$-carotene and xanthophylls in plants. Plant Cell 14:333-342.

Just, B.J. 2004. Genetic mapping of carotenoid pathway structural genes and major gene QTLs for carotenoid accumulation in wild and domesticated carrot (Daucus carota L.). PhD diss., Univ. Wisconsin, Madison, WI. Didd. Abstr. AAT 3155154.

Just, B.J., C.A.F. Santos, M.E.N. Fonseca, L.S. Boiteux, B.B. Oloizia, and P.W. Simon. 2007. Carotenoid biosynthesis structural genes in carrot (Daucus carota): Isolation, sequence characterization, single nucleotide polymorphism (SNP) markers and genome mapping. Theor. Appl. Genet. 114:693-704.

Just, B.J., C.A.F. Santos, B.S. Yandell, and P.W. Simon. 2009. Major QTL for carrot color are positionally associated with carotenoid biosynthetic genes and interact epistatically in a wild $\times$ domesticated carrot cross. Theor. Appl. Genet. 119:1155-1169.

Khachik, F., G.R. Beecher, M.B. Goli, and W. Lusby. 1992. Separation and quantification of carotenoids in foods. Methods Enzymol. 213:347-359.

Li, L. and D. Garvin. 2003. Molecular mapping of or, a gene inducing beta-carotene accumulation in cauliflower (Brassica oleracea L. var. botrytis). Genome 46:588-594.

Li, L., S. Lu, K.M. Cosman, E.D. Earle, D.F. Garvin, and J. O’Neill. 2006. Beta-carotene accumulation induced by the cauliflower or gene is not due to an increased capacity of biosynthesis. Phytochemistry 67:1177-1184.

Li, L., S. Lu, D. O’Halloran, D. Garvin, and J. Vrebalov. 2003. Highresolution genetic and physical mapping of the cauliflower highbeta-carotene gene or (orange). Mol. Genet. Genomics 270:132-138. Li, L., D. Paolillo, M. Parthasarathy, E. DiMuzio, and D. Garvin. 2001. A novel gene mutation that confers abnormal patterns of betacarotene accumulation in cauliflower (Brassica oleracea var. botrytis). Plant J. 26:59-67.

Li, L. and J. Van Eck. 2007. Metabolic engineering of carotenoid accumulation by creating a metabolic sink. Transgenic Res. 16:581585.

Lu, S., J. Van Eck, X. Zhou, A.B. Lopez, D.M. O'Halloran, K.M. Cosman, B. Conlin, D.J. Paolillo, D.F. Garvin, J. Vrebalov, L.V. Kochian, H. Kupper, E. Earle, J. Cao, and L. Li. 2006. The cauliflower or gene encodes a DnaJ cysteine-rich domain-containing protein that mediates high levels of beta-carotene accumulation. Plant Cell 18:3594-3605.
Maass, D., J. Arango, F. Wuest, P. Beyer, and R. Welsch. 2009. Carotenoid crystal formation in Arabidopsis and carrot roots caused by increased phytoene synthase protein levels. PLoS One 4:e6373.

Moehs, C.P., L. Tian, K. Osteryoung, and D. DellaPenna. 2001. Analysis of carotenoid biosynthetic gene expression during marigold petal development. Plant Mol. Biol. 45:281-293.

Okada, K., H. Kasahara, S. Yamaguchi, H. Kawaide, Y. Kamiya, H. Nojiri, and H. Yamane. 2008. Genetic evidence for the role of isopentenyl diphosphate isomerases in the mevalonate pathway and plant development in Arabidopsis. Plant Cell Physiol. 49:604-616.

Pfaffl, M. 2001. A new mathematical model for relative quantification in real-time RT-PCR. Nucleic Acids Res. 29:e45.

Pfaffl, M., A. Tichopad, C. Prgomet, and T. Neuvians. 2004. Determination of stable housekeeping genes, differentially regulated target genes and sample integrity: BestKeeper-Excel-based tool using pair-wise correlations. Biotechnol. Lett. 26:509-515.

Rasmussen, R. 2001. Quantification on the LightCycler instrument, p. 31-34. In: Meurer, S., C. Wittwer, and K. Nakagawara (eds.). Rapid cycle real-time PCR: Methods and applications. SpringerVerlag, Heidelberg, Germany.

R Development Core Team. 2011. R: A language and environment for statistical computing. 13 Sept. 2012. <http://www.Rproject.org/>.

Ronen, G., L. Carmel-Goren, D. Zamir, and J. Hirschberg. 2000. An alternative pathway to beta-carotene formation in plant chromoplasts discovered by map-based cloning of beta and old gold color mutations in tomato. Proc. Natl. Acad. Sci. USA 97:1110211107.

Santos, C., D. Senalik, and P. Simon. 2005. Path analysis suggests phytoene accumulation is the key step limiting the carotenoid pathway in white carrot roots. Genet. Mol. Biol. 28:287-293.

Simon, P.W., L.M. Pollak, B.A. Clevidence, J.M. Holden, and D.B. Haytowitz. 2009. Plant breeding for human nutritional quality. Plant Breed. Rev. 31:325-415.

Simon, P.W. and X.Y. Wolff. 1987. Carotenes in typical and dark orange carrots. J. Agr. Food Chem. 35:1017-1022.

Thorup, T.A., B. Tanyolac, K.D. Livingstone, S. Popovsky, I. Paran, and M. Jahn. 2000. Candidate gene analysis of organ pigmentation loci in the Solanaceae. Proc. Natl. Acad. Sci. USA 97:11192-11197.

Vallabhaneni, R., L.M.T. Bradbury, and E.T. Wurtzel. 2010. The carotenoid dioxygenase gene family in maize, sorghum, and rice. Arch. Biochem. Biophys. 504:104-111.

Vallabhaneni, R. and E.T. Wurtzel. 2009. Timing and biosynthetic potential for carotenoid accumulation in genetically diverse germplasm of maize. Plant Physiol. 150:562-572. 